SESSION V : Innovation en génie civil - génie côtier

\title{
La vérification des ouvrages maritimes aux états-limites
}

\author{
Jean-Bernard KOVARIK
}

Chargé de mission au Service Technique Central des Ports Maritimes et des Voies Navigables

Résumé : Les Recommandations pour le calcul aux états-limites des ouvrages maritimes, ensemble de textes élaborés avec la collaboration des nombreux auteurs (ingénieurs, chercheurs, universitaires, praticiens ...), harmonisent les règles de vérification en cohérence avec le format de sécurité des Eurocodes. On présente la structure des Recommandations et le résultat des recherches effectuées pour la définition des situations de projet, le développement d'un « cas unique » de pondération qui distingue les coefficients de sécurité à la source et les coefficients de modèle, l'harmonisation des approches « sol » et « structure » et la validation sur des projets réels.

Abstract : The texts collected into the « Recommendations for limit state design of maritime structures » include numerous contributions from engineers, research and educational institutes, praticians ... They harmonize the design rules for waterfront structures according to the safety format of the Eurocode's system. We present the layout of those Recommendations together with the investigations carried out for the definition of the design situations, the implementation of an « unique case » of partial factors, the distinction between at source factors and model factors, the harmonisation of the structural and the geotechnical approaches and the validation against real projects.

\section{Contexte}

\subsection{En France}

Le Ministère de l'Équipement a introduit au début des années 1970 le principe de la méthode semi-probabiliste aux états-limites pour la prise en compte de la sécurité dans les calculs des bâtiments et des ouvrages d'infrastructures.

De façon schématique, la mise en oeuvre de cette méthode consiste à identifier les phénomènes préjudiciables à l'ouvrage (états-limites) et à se prémunir contre leur apparition par des coefficients de sécurité (coefficients partiels) dont la valeur doit être préalablement « calibrée ».

L'instruction provisoire du 13 décembre 1971 relative aux Directives Communes pour le calcul des constructions, complétée par l'instruction technique du 9 janvier 1979 sur le même sujet [1], a été rédigée en vue de la révision des différents règlements. Son objectif était d'augmenter la durabilité des ouvrages et d'homogénéiser la sécurité de chaque partie d'ouvrage. 
Les règlements de calcul ou les codes de bonne pratique pour les bâtiments et les ouvrages d'art en béton, pour les fondations et pour les sols renforcés ont été depuis élaborés ou modernisés suivant cette approche.

\subsection{En Europe}

En 1976, la Commission des Communautés Européennes a décidé d'harmoniser les normes de calcul des ouvrages de bâtiment et de génie civil dans les étatsmembres par le programme des Eurocodes, dont le format de sécurité suit la méthode semi-probabiliste aux états-limites [2].

Actuellement, les parties générales et celles relatives aux bâtiments et aux ponts sont approuvées comme normes provisoires «ENV» (bases du calcul - béton construction métallique - géotechnique) mais il reste encore un travail important à accomplir pour d'autres catégories d'ouvrages, dont les ouvrages maritimes et fluviaux, ainsi que pour la prise en compte d'autres actions, notamment hydrodynamiques. D'autre part on a pu constater que certaines dispositions des textes ENV restaient ambiguës, en particulier pour l'application des coefficients partiels au calcul géotechnique.

\subsection{Et les ouvrages maritimes?}

Les ouvrages maritimes ont la particularité de supporter un nombre élevé d'actions variables ayant jusqu'à présent échappé à toute codification ou réglementation en France. Ils sont vérifiés en adaptant les règlements généraux en vigueur aux actions et aux conditions d'environnement particulières. De fait, on observe une certaine diversité d'approches. La pratique traditionnelle fait appel aux notions de « condition de service» et de «condition exceptionnelle » et met en oeuvre des coefficients de sécurité globaux.

\section{Les Recommandations pour le calcul aux états-limites des ouvrages maritimes}

Le Service Technique Central des Ports Maritimes et des Voies Navigables a voulu mettre à la disposition des acteurs des projets (maîtres d'ouvrages, maîtres d'oeuvre, entreprises, bureaux d'études spécialisés ...) des Recommandations pour le calcul aux états-limites des ouvrages maritimes [3] aux fins :

- de définir les conditions d'application de la méthode semi-probabiliste aux états-limites, en cohérence avec le format des Eurocodes,

- de rationaliser les règles de calcul et le choix des niveaux de sécurité,

- de préparer les professionnels aux évolutions techniques qui accompagneront l'adoption définitive des Eurocodes et leur transposition en normes françaises. 
Pour mener à bien cette entreprise, il a été nécessaire d'établir la synthèse de la pratique nationale, de mener quelques recherches spécifiques pour le développement du format des coefficients partiels et de comparer les dimensionnements obtenus avec ceux qui résultaient de l'approche traditionnelle.

Ce projet s'est déroulé de 1993 à 1998 en associant de nombreux partenaires : le comité de pilotage, comprenant des représentants des bureaux d'études portuaires civils et militaires, le réseau technique du Ministère de l'Équipement, avec la coopération duquel les recherches spécifiques ont été menées et qui a assuré la liaison avec les instances normatives européennes, des bureaux d'études extérieurs qui ont rédigé la synthèse de la pratique.

\section{Synthèse des travaux de recherche effectués}

\subsection{L'application de la théorie des états-limites}

La théorie des états-limites propose un cadre souple et un vocabulaire précis pour l'analyse des ouvrages et de leur sécurité, qui s'insère particulièrement bien dans une démarche d'assurance-qualité. Son développement a consisté en l'organisation et la classification des situations de projet et des vérifications à mener pai type d'ouvrage, définissant les phénomènes qui relèvent des étatslimites ultimes (ELU) et des états-limites de service (ELS), ainsi qu'en l'exposé des modèles de calcul appropriés. La pratique actuelle a ainsi été ré-exprimée avec la nouvelle terminologie.

La définition par le projeteur des situations de projet est un moment important $\mathrm{du}$ processus de vérification, dans lequel se posent un certain nombre de questions :

- quelles sont les différentes étapes de la vie de l'ouvrage?

- quelles sont les actions qui s'exerceront pour former les cas de charge?

- que peut-on dire des incertitudes qui affectent chaque paramètre?

- quels niveaux de sécurité semblent les plus appropriés?

Dans sa plus grande généralité, on exprime les règles de justification des ouvrages sous la forme de la vérification, selon l'équation canonique appelée «condition d'état-limite »:

Elément moteur (ou sollicitation) $\leq$ Élément résistant (ou capacité de résistance) 
Le facteur de dimensionnement $\Gamma$ a été développé en remplacement de la notion ambiguë de «sécurité de l'ouvrage ». Il est défini comme un indicateur sans dimension de la « position » de l'ouvrage pour un état-limite donné :

$$
\Gamma=\text { Élément résistant /Élément moteur }
$$

\subsection{L'application de la théorie des coefficients partiels}

\subsubsection{Le développement d'un « cas unique » de pondération}

Dans l'approche semi-probabiliste, les paramètres du projet sont assimilés à des variables (ou à des processus) aléatoires. Les diverses valeurs possibles des paramètres sont appelées «valeurs représentatives ». Le projeteur définit d'abord la «valeur caractéristique » par un fractile donné ou une période de retour dans la distribution statistique supposée du paramètre ; cette valeur est utilisée principalement dans la combinaison rare des ELS. La « valeur de calcul », utilisée dans la combinaison fondamentale des ELU, est (le plus souvent) déterminée par l'application d'un coefficient partiel sur la valeur caractéristique : la valeur du coefficient partiel tient alors compte de la variabilité du paramètre, de son influence dans la condition d'état-limite et du niveau de sécurité requis.

La notion de valeur caractéristique appliquée aux propriétés des sols a été précisée dans le but de concilier la définition statistique avec la pratique géotechnique qui fait largement appel à l'expérience.

Les cas de pondération $« \mathrm{~B} »$ et $« \mathrm{C} »$ des Eurocodes traduisent respectivement des approches «structure » et «sol », selon que l'on préfère pondérer avant tout les actions (B) ou les propriétés des sols (C). Il est apparu que, s'agissant d'actions dues au sol, les paramètres géotechniques intervenant aussi bien du côté des actions que des résistances, la prise en compte de la sécurité devait être différente selon la nature de l'action (pressions en poussée et butée, actions purement gravitaires, actions liées à un déplacement d'ensemble du sol). Pour harmoniser les cas $B$ et $C$, il a été choisi de développer l'usage encore peu répandu des coefficients de modèle. Les coefficients partiels sont regroupés en deux séries :

- les coefficients partiels qui représentent l'incertitude afférente à la valeur des paramètres auxquels ils s'appliquent, quel que soit l'étatlimite que l'on cherche à vérifier : on les appelle alors « coefficients à la source »,

- les coefficients de modèle notés $\gamma_{d}$ selon l'ISO 2394 (notés $\gamma_{F 3}$ dans le CCTG, $\gamma_{\mathrm{rd}}$ et $\gamma_{\mathrm{sd}}$ dans les Eurocodes) qui représentent l'incertitude causée par la mauvaise adéquation entre les résultats d'un modèle de calcul et la réalité du comportement des ouvrages. 
Les coefficients de modèle sont définis pour chaque état-limite et introduits conventionnellement dans la condition d'état-limite par :

$$
\gamma_{d} \text {. Élément moteur } \leq \text { Élément résistant }
$$

Les éléments moteurs et résistants sont calculés quant à eux avec les coefficients «à la source ».

On constate que les cas $\mathrm{B}$ et $\mathrm{C}$ s'assimilent à des cas particuliers de cette nouvelle formulation.

\subsubsection{Le calage des coefficients partiels}

Pour établir les valeurs des coefficients à la source, l'on s'est basé sur les textes existants (BAEL, fascicule 62 titre V, TA 95, CLOUTERRE 91) dont on a essayé de reprendre les valeurs autant que possible.

Une démarche spécifique de calage des coefficients de modèle a dû être mise en oeuvre par type d'ouvrage (soutènements-poids, soutènements-plan, barrages mobiles, ducs d'Albe souple, ouvrages sur pieux ...).

Le calage a consisté à répartir la sécurité entre les coefficients à la source préétablis et les coefficients de modèle, de sorte «qu'en moyenne» les dimensionnements restent raisonnablement proches des dimensionnements traditionnels.

\subsubsection{Quelques applications expérimentales}

La validité de la nouvelle approche a été testée sur quelques projets réels. On a pu ainsi effectuer quelques exercices où l'ensemble de la démarche a été mise en oeuvre: barrages mobiles de Diama, Montmeuse, Saint-Albin [4], projet d'appontement sur pieux de Cordemais, ducs d'Albe d'accostage et d'amarrage des postes rouliers et pétroliers de Donges et de Montoir, quai en bloc à la Ioliette, rideau de soutènement ancré à Dunkerque [5 et 6].

Ces études ont démontré que la nouvelle approche était aisée à mettre en oeuvre et qu'elle donnait des résultats acceptables.

\section{Recommandations: Mode d'emploi}

\subsection{Contenu}

Les Recommandations établissent les raccordements avec les textes généraux en vigueur, notamment le BAEL et le fascicule 62 titre $\mathrm{V}$. Elles traitent de la sécurité dans toute la chaîne des calculs selon l'approche décrite ci-dessus, mais 
n'abordent la conception et l'exécution des ouvrages que dans la mesure nécessaire à l'intelligence de leurs règles de justification. Elles ne traitent pas des questions relatives à la qualité des travaux.

\subsection{Utilisation}

Au moment de la conception : bien que celle-ci soit hors du champ principal visé par le texte, les Recommandations présentent des indications générales qui peuvent être utiles à ceux qui ne sont pas familiers des ouvrages maritimes.

Au moment de l'avant-projet : on engage la réflexion sur les situations, les phénomènes préjudiciables à éviter, le caractère favorable ou défavorable des paramètres divers, les modèles de calcul ...

Au moment du projet : l'optimisation de l'ouvrage fait appel à un nombre très élevé de cas de charge et de combinaisons d'actions dont la mise en oeuvre n'apparaît raisonnable qu'avec l'aide d'un logiciel de calcul automatisant les entrées/sorties. Pour contourner cette difficulté pratique de mise en oeuvre, inhérente au format semi-probabiliste aux états-limites, il y a lieu de sélectionner a priori un nombre réduit de combinaisons d'actions et d'états-limites pour effectuer les vérifications, en se fiant à l'expérience et à quelques calculs préliminaires.

\subsection{Structure}

Les Recommandations sont divisées en fascicules thématiques regroupés par séries.

\subsubsection{Dispositions communes}

Les fascicules de la série Dispositions communes s'ouvrent par une présentation d'ensemble où le format semi-probabiliste aux états-limites, le cheminement concret des vérifications et les règles de classification des ouvrages selon leur importance sont décrits. On propose ensuite des règles pour :

- la détermination des situations de projet et l'écriture des combinaisons d'actions (avec les coefficients d'accompagnement $\psi_{0}$ ),

- la détermination des cotes en pied d'ouvrage, la prise en compte de la corrosion, les critères imposés pour certains états-limites de service,

- la détermination des propriétés de base des sols, du béton et de l'acier, des propriétés de résistance des pieux, des tirants, des fondations superficielles et des éléments de structure, des paramètres d'interaction sol-structure (courbes de réaction, paramètres de frottement ...), avec les coefficients partiels y afférent. 
- talus et pentes, sols renforcés,

- parties en béton des ouvrages.

\subsubsection{Documents d'application}

Les fascicules de la série Documents d'application présentent des outils de mise en oeuvre et quelques exemples pédagogiques:

- guide pour la rédaction du chapitre «Hypothèses de calcul» des C.C.T.P.,

- canevas-type pour les notes de calcul de vérification,

- exemples commentés de notes de calcul d'ouvrages : situations et combinaisons d'actions, appontement sur pieux, quai en blocs, rideau de soutènement ancré, ducs d'Albe souples.

\section{Conclusion}

Les Recommandations pour le calcul des ouvrages maritimes aux états-limites forment un ensemble cohérent qui exprime les règles de sécurité selon la méthode semi-probabiliste aux états-limites. Établies en conformité avec le format des Eurocodes, elles simplifient l'usage des cas de pondération en étendant les règles du BAEL et du fascicule 62 titre $\mathrm{V}$ à toute sorte d'ouvrage.

L'avantage majeur du nouveau format de vérification est d'identifier plus clairement quelles incertitudes l'on traite et de quelle façon, en recherchant toujours l'homogénéité des approches pour les diverses parties d'ouvrage.

De nombreux calages de coefficients partiels de modèle ont été réalisés. Le niveau de sécurité général obtenu, proche en moyenne du niveau de sécurité antérieur, doit continuer d'être examiné dans les projets réels. Le retour d'expérience est ainsi vivement attendu.

\section{Remerciements}

Ce projet a été financé par le Ministère de l'Équipement (Direction du Transport Maritime, des Ports et du Littoral - Direction des Transports Terrestres) et à bénéficié du Fonds d'Investissement des Transports Terrestres et des Voies Navigables. 


\subsubsection{Equipements}

Les fascicules de la série Equipements donnent des indications utiles préalables au calcul de certaines actions. Ils concernent les outillages portuaires et les défenses d'accostage pour lesquelles des règles de vérification sont proposées en cohérence avec le format de sécurité retenu pour les infrastructures.

\subsubsection{Actions}

Les fascicules de la série Actions proposent des règles pour la détermination des valeurs représentatives des actions, avec les coefficients partiels et les coefficients $\psi_{1}$ et $\psi_{2}$ y afférents :

- actions de l'eau : détermination des niveaux d'eau des fleuves, des bassins portuaires, dans les terre-pleins, dans les massifs confinés, avec la prise en compte des surcotes et des décotes et le calcul des gradients d'écoulement (action quasi-statique des niveaux d'eau et la carte des niveaux extrêmes de la mer en Franice), action du courant, action de la houle,

- actions du sol en poussée et butée, actions liées à un déplacement d'ensemble (poussées latérales, frottement négatif), forces d'écoulement, transmission d'actions par le sol chargé, action pondérale du sol (regroupées sous l'appellation « actions du terrain »),

- accostage, amarrage, détermination des charges roulantes du trafic ordinaire et spécialisé, descentes de charge des outillages portuaires fixes et sur rails, charges de stockage réparti sur les terre-pleins (charges d'exploitation),

- actions accidentelles : séisme, tsunami, choc de navire contre un obstacle fixe, configurations accidentelles (rupture de tirant ...).

\subsubsection{Ouvrages}

Les fascicules de la série Ouvrages présentent les états-limites à vérifier avec leurs modèles de calcul, indiquent les valeurs des coefficients de modèle :

- structures gravitaires à fondations superficielles : quais en blocs, caissons, ducs d'Albe rigides, gabions de palplanches,

- rideaux de palplanches et parois moulées, ancrées ou non,

- ouvrages à fondations profondes : appontements quais sur pieux, ducs d'Albe souples, 


\section{Références}

[1] Directives Communes de 1979 sur le calcul des constructions - Ministère de l'Équipement.

[2] norme P 06-101-1 ENV 1991-1 et DAN : Bases du calcul - AFNOR.

[3] Recommandations pour le calcul aux états-limites des ouvrages maritimes (avril 1998, version C, soumise à l'enquête) - STCPMVN, Ministère de l'Équipement.

[4] Alain CARRERE et Michaël COLSON (1997) - Mise au point d'une méthode semi-probabiliste aux états-limites pour le dimensionnement des barrages mobiles - étude réalisée par Coyne et Bellier pour le compte du STCPMVN.

[5] Cyril BONNET (1997) - Dimensionnement aux états-limites d'ouvrages maritimes de soutènement et d'un appontement sur pieux - Service Technique Central des Ports Maritimes et des Voies Navigables - École Nationale Supérieure des Mines de NANCY (rapport de stage).

[6] Stéphanie LINGLIN (1997) - Dimensionnement aux états-limites de ducs d'Albe d'accostage et d'amarrage - Service Technique Central des Ports Maritimes et des Voies Navigables - Institut National des Sciences Appliquées de LYON (rapport de stage). 